\title{
THE "HALLIWICK" SWIMMING METHOD FOR THE HANDICAPPED (Adults and Children)
}

\author{
Developed by the Association of Swimming Therapy U.K.
}

\author{
A. C. Lochau (Physiotherapist (R.N.))
}

\section{Introduction:}

At the opening of the Selwyn-Segal Home for Jewish Handicapped, in Sandringham, Johannesburg, by the Honourable, the Administrator" of the Transvaal, Mr. S. G. van Niekerk, he pertinently referred to the growing realisation that many handicapped persons in South Africa could become useful citizens by applying special therapies. He stated that, in 1965 , there were some 2000 educable handicapped in the Republic and noted with regret that there were only nine schools for the handicapped. Rather more schools or institutions were urgently needed to provide the specialized treatment he had referred to earlier in his speech.

Attention is given to the handicapped in most countries. Schools or homes have been provided by the State, Churches and welfare societies and other assistance is frequently given to help the handicapped and to assist them to become as independant and as useful to the community as possible. In Switzerland, for example, the Swiss Social Invalid Insurance provides both young and older handicapped persons with the opportunity to visit Spa Clinics annually to receive special free treatment.

All this work is highly commendable, yet it cannot entirely overcome the position that the handicapped person largely lives in a world apart. His is the world of appliances and therapies. Especially where he is one of a family of normally developed children, the handicapped child becomes aware of his deficiencies, as participation in most games and sport is usually impossible. Therefore a feeling of isolation can easily develop with consequent enduring physical and psychological effects.

Considering these facts, Mr. J. McMillan, a friend of the Halliwick School for the Handicapped run by the Church of England's Children's Society and accommodating mainly Cerebral Palsied children, conceived the idea that it might be possible to largely overcome this disparity by inducing the children to participate in water sports, that is, swimming or playing games or merely dis porting themselves in water. The emphasis was to be placed on the recreational aspect, the pleasure derived from this sport, although, of course, the therapeutic value may not be underestimated.

The idea was discussed with the School's honorary surgeon, Mr. O. J. Vaughan Jackson, F.R.C.S., who gave it his wholehearted support.

The sponsors of the scheme were allowed to proceed and in 1950 twelve girls in the age group 9-15 years were elected for the first course. The experiment was started with enthusiasm, but it was realised very soon that the methods adopted to teach normal children to swim, had comparatively little value in this case. An entirely different approach appeared to be necessary. In the ensuing months most attention was therefore devoted to devising suitable teaching methods. Thereafter con- siderable success was attained, more children participated and the "Halliwick Penguins" Swimming Club could be established at the school.

World-wide interest in the scheme was created by $\mathrm{Mr}$ ? Bobarth who visited the school, studied the method and published her findings. Thereafter physiotherapists from all over the world visited the school and disseminated the idea in their countries. However, the most rapid development occurred in the United Kingdom. There, other clubs for handicapped people adopted the ideas and soon so many clubs were formed that inter-club galas could be arranged. In fact, the supporters of this new approach found it advisable in 1952 to establish the "Association of Swimming Therapy" in the United Kingdom. The objects of the Association are: to act as the central co-ordinating organisation, to frame a uniform code of practice and to act as clearing house for the collection and dissemination of the experience gained, to strive to improve methods and to devise new methods or games to help the handicapped.

The present description of the method is based, mainly, on a course given by Mr. J. McMillan and Dr. Joan Martin, as guest lecturers at the Swiss Spa-Clinic, Bad Ragaz during the spring of 1965 , which the present author was privileged to attend. The course consisted firstly of lectures and practical demonstrations and later the participants to the course had the opportunity to practise the method, under guidance, on a group of adult handicapped who visited Bad Ragaz by arrangement with the Swiss Invalid Social Insurance.

In this discussion the instructor or trainer will generally be referred to as "helper" and the handicapped person ah "swimmer" irrespective of whether he actually learns to swim, or only to move or disport himself in water.

\section{Prerequisites for success as Helper:}

In order to understand and successfully apply the method, the helper must first have a fair knowledge of physics, especially matters such as force, moments and levers, stress and strain, the effects and force of gravity, balance, the properties of water and their effects on submerged bodies, e.g. pressure, buoyancy and of the theorems relating to fiuids, such as those of Archimedes and Bernoulli. Attention should be given to the effects of water on the normal as well as the handicapped human body.

Secondly, the helper must have knowledge of the human body, its anatomy and physiology and in particular of the neuro-muscular system. In this context the helper should also study the mental aspect of the handicapped and his probable reactions when transferring from the medium air, to which he has become used to, to the denser medium, water. 


\section{The Swimmer's Aspect:}

The human body is constantly subject to the force of The hund has to react to its effect when changing shape gravity anding movement through muscle action. In the or maint" medium, air, these reactions have become subconscious. Similarly breathing is a subconscious action in the medium air.

All this naturally also applies to the handicapped, Asually assisted in maintaining posture or motion by various types of appliances. In his case, however, there is the experience of inability for action without the is the expe of the particular appliance and a greater sense assistance falling and hurting himself. Since, for reasons of fear later, these appliances are to be abandoned when the swimmer enters water, the helper must be very conscious of this element of fear in his pupil.

The force of gravity still reacts on bodies partly or wholly submerged in water, but in this medium of much higher density than air, the body experiences a very appreciable upthrust acting in a direction opposite to that of the force of gravity. Since the human body has average density lower than that of water, the relaxed fody will actually float in water, albeit in more or less horizontal rather than upright position.

A person entering deep water thus has less appreciation of the force of gravity, indeed most of the fixed points (relative to the medium air) appear to be lost.

As is well known, water, at any point below the surface, exerts pressure equally in all directions, the pressure rising with depth from the surface. The human body, submerged in water, therefore experiences much higher pressure against the chest than in air and the subconscious pattern of breathing in the medium air is affected. A new pattern of breathing must therefore be developed.

This loss of the acquired fixed points (in air) and the hampering of breathing add to the sense of fear or insecurity of the handicapped person. One may of course add to this the fear arising from gulping or choking in water by inadvertently getting water into the mouth or nose. The result of fear is tension which is detrimental to proper movement in water. The first object of training is therefore to overcome this fear, and to teach the swimmer to enjoy being in the water.

This can be achieved both consciously and subconsciously by devising various games to be played in the water. The swimmer progressively becomes used to water and its forces, such as the balance between gravity and buoyancy. By learning to swim in the supine position first, to float and to recover to a safe breathing

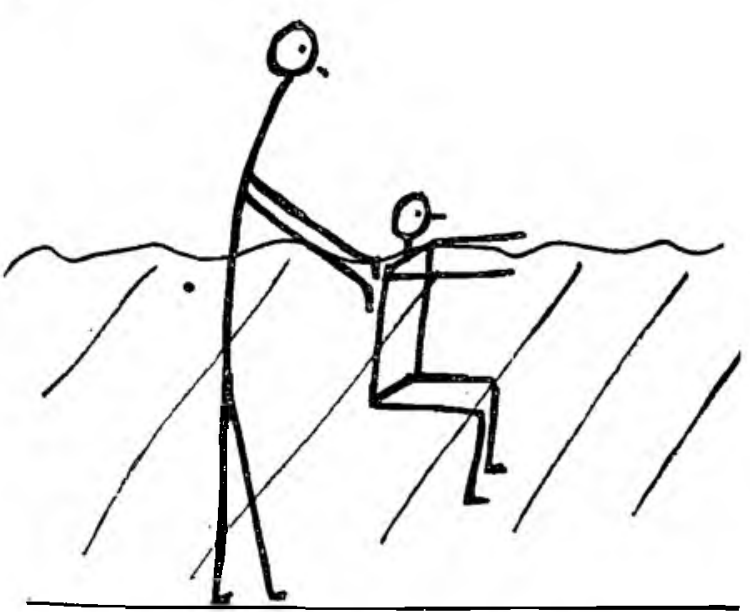

Figure 1. "Sit on your stood." position, the swimmer gains the necessary confidence for independent swimming.

The two mottos of the method are therefore:-

(1) BALANCE IS STILLNESS!

(2) BLOW1

\section{The Application of the Method:}

It must be stressed at the outset that the underlying principle of the method is to provide the handicapped with an opportunity to enjoy himself freely. The idea should not arise that this is another form of therapy. There must therefore be no form of compulsion. The handicapped should participate quite voluntarily and the swimming should be made as normalised and enjoyable as possible.

In order to achieve this, no appliances, neither those used normally by the handicapped or so-called swimming aids are allowed in this method. The swimmers are lifted onto the side of the pool - entry is from the side of the pool, the helper always close by the swimmer to remove all sense of fear of falling. The helper may assist at first, both in entering and getting out of the pool

The dissipation of fear is also achieved by assuring the swimmer that he cannot be hurt as when falling on land. Drowning need not be feared as the helper for each swimmer is always close by. Furthermore the fear of drowning is removed by telling the swimmer that if he relaxes and becomes completely still, the water will push him to the surface: "You cannot stay under, even if you want to."

The swimmer should also be taught from the outset to BLOW when his nose or mouth comes near to under the water.

During the training, established ideas from life on land are used. For example, to acquire head control the swimmer is told: "Sit on a stool, place your hands on the table." (He then "sits" till his shoulders are submerged - being lightly supported by the helper's hands on his shoulder-blades. See the sketch.) "Now lie back on your bed and sleep." The helper (each swimmer must, at least in the initial stages, have his own helper) is always behind or at the side of the swimmer after he has entered the water. When behind the swimmer, he lightly places his hands on the swimmer's shoulder-blades. He does not hold the swimmer's head as it must be free to move to be able to learn proper head control).

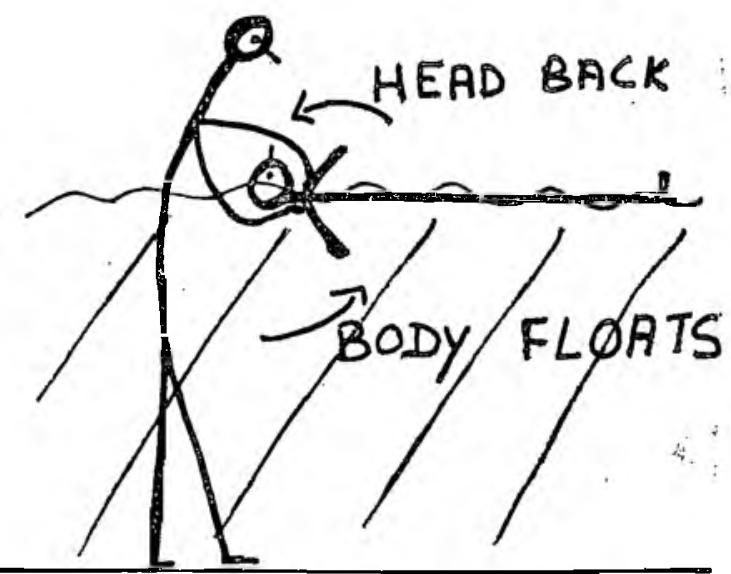

Figure 2. "Lie down on your bed." 


\section{Enjoy the South African way of life}

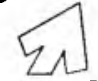

Growthowith Security

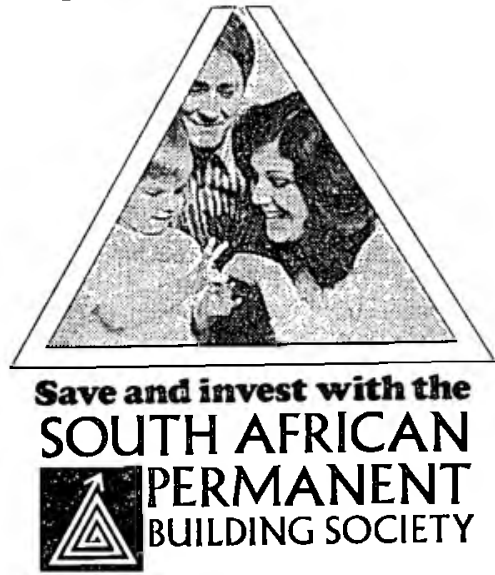

Grant 2467/3

\section{A. C. MILLER \& CO. ORTHOPAEDIC MECHANICIANS}

Technicians registered with S.A. Medical and Dental Council specialising in the following: ORTHOPAEDIC APPLIANCES, SURGICAL CORSETS, CERVICAL COLLARS, CHILDREN'S SHOES AND BOOTS, ARTIFICIAL LIMBS, LATEST IN PLASTIC MODIFICATION. HIRING AND SELLING OF HOSPITAL EQUIPMENT AND SICK ROOM REQUISITES, e.g. WHEEL CHAIRS, COMMODES, HOSPITAL BEDS, WALKING AIDS, TRACTION APPARATUS, etc.
Telephone 23-2496
P.O. Box 3412 275 Bree Street
Johannesburg

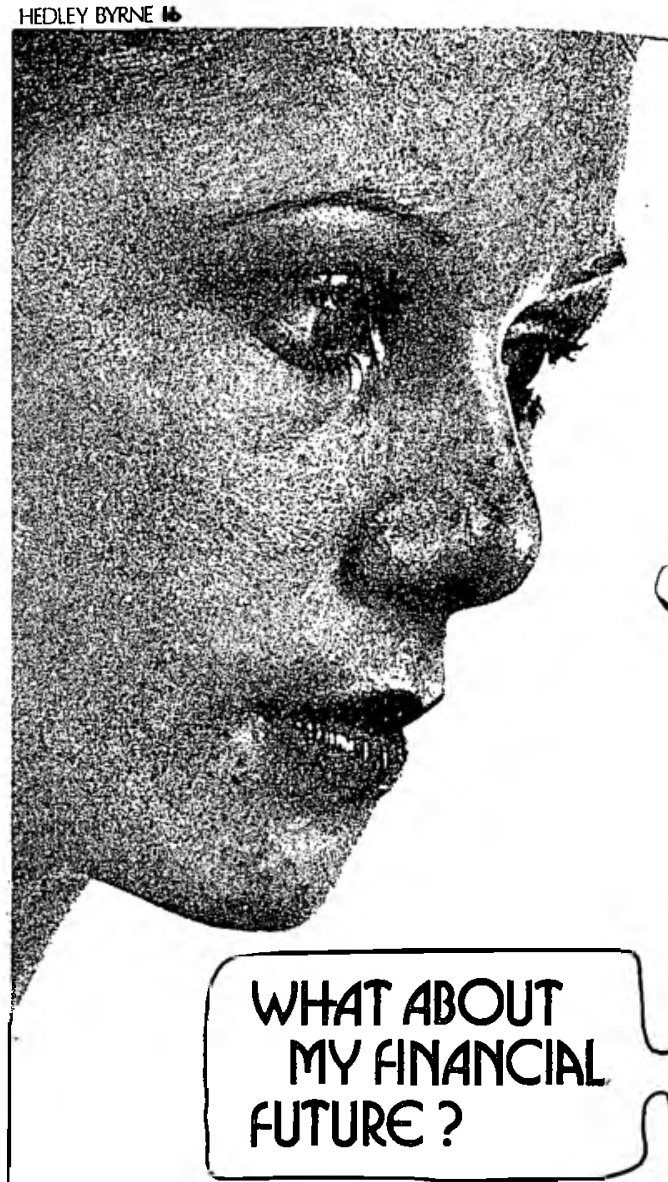

CROUP INSURANCE offers you special benefits at reduced rates including valuable disability benefits.

Your financial future is more secure when you join YOUR SWN ASSOCIATION'S Superannuation Fund. It costs you less to insure the "Group" way

Fill in and post the coupon below for full particulars of how ( you can benefit from your S.A. NURSING ASSOCIATTON'S GROUP INSURANCE SCHEME-all correspondence will be treated in strict confidence.

To the Trustees, S.A. Society of Physiotherapy, Group Endowment Fund, P.O. Box 1194, Johannesburg.

Name

Address

Age next birthday

Occupation

Where employed

How much can you afford to contribute each month?... 
The teaching must always be positive. All the orders musi also be positive - never use the word "Don't". musi also be the teaching aims at the development of Throughout, thol and the maximum use of water's buoybalance, control and the mas, even if often rather slowly, mental and ahysical security develop.

Then, and then only!, proper swimming and diving lessons may be started. (As indicated already, "swimming" means any possible method that the swimmer may ming to propel himself independently from one side of the pool to the other, and his ability to regain the standing or a safe position by himself.)

It is essential to exercise great patience - never rush swimmer, even if it may take years to accomplish one step forward. It is better to spend twenty lessons on one small point than to try to rush a "slow" swimmer to keep up with the group's advance.

Although it may be advisable to take a new swimmer to the pool separately for the first two or three sessions, in order to assess his ability and emotional behaviour, the teaching is done in groups. The number of parcipants in a group depends on the size of the available swimming pool (or the part of it having a reasonable depth, i.e. where the helper can stand firmly and the swimmer may touch the floor when recovering to a standing position) and the number of helpers available. It should also only be large enough to enable the leaderhelper to exercise control of the whole group at all times. For example, the swimming pool at the Pretoria School for Cerebral Palsy (size approximately 15 feet by 20 feet) could only accommodate 4-5 swimmers as well as their helpers (thus about 8-10 in each group). One of the helpers acts as leader of the group.

The final grouping of swimmers is not done according to age or disability, but rather according to each individual's ability in the water. The standard of ability may not be very obvious, but it is essential that the members of each group of swimmers should have approximately equal ability.

Each group has one leader, who controls the activities generally and gives the orders. The leader usually remains constant. The helpers ensure that their respective swimmers participate in the activities and overcome their own specific problems. The leader should be changed frequently, as well as the different helpers, so to avoid emotional attachment by a swimmer to a particular helper. There are of course exceptions to this rule, as in cases of difficulty of communication (e.g. deaf or blind swimmers and bad cerebral palsied swimmers who have ifficulty in talking).

Teaching is done mainly by playing games similar to those played by normal children on land. Such games can be given the same names, e.g. "Apples and Oranges", "Cat and Mouse" and "Ring a Ring a Rose". Each game has some specific objective. The swimmers may gain this point subconsciously or, when it appears desirable, they may be informed what is to be achieved.

Joyous participation is encouraged. Lots of shouting and laughter help to relax the swimmers, the co-operation improves and a competitive atmosphere usually develops. In this way most of the important lessons may be learnt.

Instruction, through such games, is given in breathing, head control, standing, walking, jumping and swinging in static positions (aided by two helpers) - for subtle head control - forward recovery (to come to a standing position from supine lying) and rolling recovery (to obtain a safe breathing posture in the supine position).

Other lessons are given to create underwater confidence as a preliminary to independent entry into the water. These include practice in somersaulting and rolling. Finally lessons in pushing off, gliding, sculling with turns, backstroke and other strokes are steps towards the objective of independent swimming.
It is very essential that progress should be made. Since this may be very slow, the helper must have infinite patience. Furthermore, it cannot be stressed suffciently that facilities should be available for regular training of swimmers, especially for the beginners, throughout the year. Much valuable work may become completely undone if the training has to be suspended for long periods - due, for example, to cold or rainy weather.

The provision of incentives to ensure sustained progress is valuable. Praise and encouragement from the helper should never be lacking. Small prizes and provision of grades, marked by a ribbon or badge have proved useful.

The training of people, who after leading a normal life, including for example water sports, have become handicapped through some accident or disease, presents special problems. Many of these people acquire a fixed idea that they will never be able to use their limbs again, and accordingly they are very sceptical when exercises in water and swimming are suggested to them. They are generally more afraid of entering the water than other handicapped persons. However, if they can be persuaded to enter the water and learn again to swim independently they are generally more grateful and a lot happier about the achievement than other handicapped persons, who were born with their disability.

\section{Practical Experience Gained:}

The task may seem, even from this brief description, to be formidable. The potentialities of the method were, however, illustrated pertinently during the demonstrations at Bad Ragaz, mentioned in the introduction, where only the leader was entirely conversant with the course.

The trainee-helpers had only eight sessions of about one hour each with a group of sixteen to twenty invalids. During this short period more than half of the swimmerpupils learnt to swim independently fairly well. Among them was a particularly sceptic young paraplegic, who had been able to swim before. his accident and would not believe that he could be trained to swim again. Another successful swimmer was a very rigid cerebral palsied quadriplegia (18 years old), who had never swum before. This young man had only very slight head movements (about $25 \%$ ) and only about $15^{\circ}$ knee and shoulder movements. Yet he learnt to go into the supine position by himself, could swim across the pool and pull himself into the standing position. It was only at this juncture that he had to be helped to remain standing.

At the School for Cerebral Palsied in Pretoria, there has not yet been much opportunity to practice the method as the pool can only be used during the summer months, provided it does not rain. The water is cold, and especially those children who are badly disabled get very cold, tense and shivering too soon. Also the cold water may be very unpleasant for the helpers who are virtually static. The helpers should keep the shoulders in the water. The few children who could be helped were as astonished and pleased as their therapists at their own progress achieved.

\section{Application of the Method and Progress in U.K.:}

As the application of the method is probably most advanced in the U.K. a brief description of developments under the auspices of the Association of Swimming Therapy may serve to indicate the possibilities.

\section{Clubs:}

The prime object of the method is to create as normal an atmosphere for the handicapped as possible. Clubs may be formed as soon as a large enough group of participants is available. Each Club is given a name which specifically should have no reference to the fact 
that the swimmers are handicapped persons. Typical names are:-

Kensington Emperors Swimming Club.

Halliwick Penguin Swimming Club.

Starfish Swimming Club.

Crewe Seahorses Swimming Club.

Membership of a club gives the swimmer the very valuable feeling of "belonging". This is accentuated by the club badge.

\section{Rules and Membership:}

A short set of rules is necessary for proper order. The Association has found that this can be confined to about twelve rules.

Full membership is only available to handicapped people. Helpers, parents and others prepared to help or support the club in any way, may become associate members.

\section{Management and Stafi:}

The club is usually managed by a committee consisting of a President, Chairman, Secretary and Treasurer (or Secretary-Treasurer) who are "normal" people of responsibility.

The "staff" consists of a chief instructor assisted by senior and junior (assisting for example in dressing, grouping and preparing and serving refreshments, as well as transport officers (from and to the club and homes). None of the participants (swimmers, instructors or helpers) wear any uniform and to ensure anonimityall are only known by their Christian names. All "posts" are honorary and staff candidates are accepted, not by reason of the fact that "they may be sorry for the poor little children" but only if they have a genuine desire to help those less fortunate than themselves; finding their reward in the joy they bring to the handicapped and the satisfaction arising from unstinted service. Many assistants come from the ranks of parents and of organisations such as the St. John's Ambulance, the Trefoil Guild, the B.P. Guild, the Girl Guides and Boy Scouts. These mainly assist the swimmers with undressing and dressing, drying of swimmers, as vehicle drivers and acting as escorts on transport vehicles. Some become instructors assisting the trained instructors.

\section{Subscriptions and Funds:}

As stated above, all assistants give their services free of charge. Swimming pools are either provided specifically for this purpose by local authorities or public baths made available free for pre-determined periods. Local authorities also provide free transport for swimmers and their assistants while organisations such as the Rotary Clubs or the Round Table also provide transport facilities. Many firms also assist by, for example, donating refreshments.

The actual club expenditure is therefore quite moderate. Nevertheless, there is unavoidable expenditure and it is also considered that a moderate club fee adds to the feeling of really "belonging" to a worthwhile organisation. Therefore, full senior members pay an annual membership fee of about 25 cents while juniors pay 15 cents per annum. The associate members' fee is 25 cents per annum. Donations are kept in fund for arising necessities.

\section{Tests:}

The Association has laid down a series of tests which swimmers must pass for promotion from one grade to another. Some 24 steps of progression are recognised and a badge or insignia is provided for each grade.

\section{Inter-Club Activities:}

While the provision of generally recognised grades stimulates the swimmer to individual effort and helps to build up confidence not only in the swimming pool but, probably subconsciously in all spheres of his activities, it was recognised that much social benefits could accrue by arranging inter-club (i.e. if necessary inter-school) meetings or galas.

On the one hand a normal club or school-spirit evolves. On the other hand, bearing in mind that the handicapped children hear so much about inter-school galas from their normal brothers, sisters and friends, that the institution of similar galas tends to give the whole club movement a more "normal" atmosphere, as well as adding to the prestige of the handicapped in their family circles.

All races at galas are swum on a time-handicapped basis, as this is the only way to give a fair chance to every competitor. Thus if four swimmers normally swim 30 metres in:

\section{A: 30 seconds B: 35 seconds $C: 65$ seconds $D$ : 90 seconds}

then D will start at the signal "Go!" while C, B and will follow at the required intervals, so that all would "normally" end the race at the same time. In fact, a swimmer is usually not allowed to beat his "normal" time by more than one second per ten yards.

These galas have become very popular and are well supported.

\section{Requirements for Applying the Method in South Africa:}

For training purposes a pool of about $20^{\prime} \times 40-50^{\prime}$ having a depth of about $3^{\prime}-3^{\prime} 6^{\prime \prime}$ would be adequate. (Bigger pools allow bigger groups or more groups.) It is preferable to have the pool closed in and provision for maintaining the water temperature at about $80-85^{\circ} \mathrm{F}$ $\left(26-29^{\circ} \mathrm{C}\right)$ is necessary. The pool, or at least about one third of it, if it is a large pool, should be reasonably shallow to allow the helper to be able to stand easily during all the training sessions. The temperature should be reasonably high constantly to enable training to be continued throughout the year, secondly because the helpers remain in the water for quite extensive periods without being very active, and thirdly because of the rather poor circulation as well as the inability to be very active often, of the handicapped, so that they get cold very quickly.

While it is helpful to have such specific pools, good use can be made of the shallow ends of large pools, the deeper ends being used for the more independent swimmers. Since it is being more generally realise that baths suitable for all the year use by normal swimmers are becoming a necessity to ensure the maintenance and even improvement of the high standard of South African swimmers (Transvaler 21.7.67), it can be sincerely hoped that such baths will soon be provided at all important centres in the Republic and that these can then also be made available to the handicapped at certain times.

Surely, local authorities, other organisations and also individuals would be prepared to assist to provide transport and other assistance to launch such a scheme. One would have to start with a few clubs only at first, but in areas such as the Witwatersrand-Pretoria complex, Cape Town and environs, Port Elizabeth and Durban inter-club meetings might be instituted in due course.

In this article stress has been laid on the recreational aspect. The therapeutic effect both physically and mentally cannot be overlooked, however. Therefore the institution of the scheme should also be of great value in achieving the objective mentioned by the Administrator in his speech, of striving to develop all the potentialities of the handicapped so that the maximum number possible may become independent and useful members of the community. 